\title{
"So young, and so untender? " : le don du père à la jeune génération dans Le Roi Lear
}

Anne-Kathrin Marquardt

\section{(2) OpenEdition}

\section{Journals}

\section{Édition électronique}

URL : http://journals.openedition.org/shakespeare/3705

DOI : 10.4000/shakespeare.3705

ISSN : 2271-6424

Éditeur

Société Française Shakespeare

\section{Référence électronique}

Anne-Kathrin Marquardt, « «So young, and so untender? » : le don du père à la jeune génération dans Le Roi Lear », Actes des congrès de la Société française Shakespeare [En ligne], 34 | 2016, mis en ligne le 01 mars 2016, consulté le 21 avril 2019. URL : http://journals.openedition.org/shakespeare/3705 ; DOI : 10.4000/shakespeare.3705

Ce document a été généré automatiquement le 21 avril 2019.

(c) SFS 


\title{
"So young, and so untender?": le don du père à la jeune génération dans Le Roi Lear
}

\author{
Anne-Kathrin Marquardt
}

1 Quelles sont les obligations des jeunes face aux plus âgés? C'est la question qui est au cœur du Roi Lear, pièce qui met en scène un conflit intergénérationnel. Ce différend se nourrit notamment du problème de l'héritage, posé dès la scène d'exposition avec le don du royaume du père aux enfants. La façon dont les personnages interprètent leur rapport aux choses, notamment aux choses données, échangées ou refusées, offre une perspective sur les rapports qu'ils entretiennent entre eux. Quelle est la légitimité d'une jeunesse qui s'en tient souvent à une vision matérialiste des rapports humains, face à une vieillesse qui impose des obligations absolues?

\section{«I gave you all »: Lear ou l'obligation absolue}

2 Le «don» de Lear (I.i.35-140) pose plusieurs problèmes. Premièrement, la nature de l'opération n'est pas clairement définie : don, contrat ou héritage anticipé ? Si nous nous penchons d'abord sur la question de l'héritage, il s'agit d'une forme de don particulier, survenant après le décès du donateur, et qui consiste à abandonner tous ses biens à un autre. Cela peut d'ailleurs susciter un sentiment de culpabilité et d'endettement pour le donataire, qui ne saurait rendre cet immense don au défunt ${ }^{1}$, car par définition l'héritage ne peut être remboursé. Mais comme Lear lègue justement ses biens de son vivant, un remboursement est tout à fait possible. Si nous interprétons le geste de Lear comme un legs ${ }^{2}$, alors il combine la force de l'héritage, qui endette totalement, avec la possibilité pour le roi d'exiger un retour. C'est peut-être ce qui explique que Lear lui-même ne présente pas la cession comme un legs; il la décrit tantôt comme un contrat, tantôt comme un don, c'est-à-dire deux formes de passation de la propriété qui lui permettent d'exiger un remboursement. 
3 Le geste ressemble à un contrat en ce que Lear stipule clairement sa part de l'accord, les choses qu'il cède. Cela comporte la " gestion » quotidienne du royaume, le pouvoir et les ressources financières, la pompe royale, et bien sûr les terres elles-mêmes. En échange, Lear s'attend à recevoir des contreparties, définies tout aussi clairement : la déférence due au roi, les cent chevaliers que les enfants devront nourrir et loger, avec le vieux roi, à tour de rôle. C'est ainsi dès le premier acte que Lear inaugure l'obsession de la quantité qui traversera toute la pièce en émaillant son discours de comparatifs et de superlatifs :

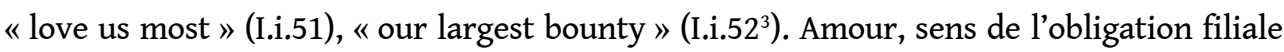
et biens matériels sont mis sur le même plan; tous peuvent être comparés et par là quantifiés.

4 Pourtant, Lear utilise également le langage du don pour décrire son geste. La terminologie est assez explicite quand Kent demande à son souverain : « Revoke thy gift » (I.i.165). Lear souligne sa propre générosité : «so kind a father!» (I.v.31). Il mentionne souvent l'ingratitude qu'il croit observer chez ses filles aînées, insistant sur un lien d'affection entre les générations plutôt caractéristique du don: "this tempest in my mind / Doth from my senses take all feeling else,/ Save what beats there, filial ingratitude » (III.iv.12-14). Les registres de l'échange de contreparties et celui du don gratuit sont ainsi mélangés dans le discours du roi.

5 Le second problème dans le geste de Lear, c'est que le récipiendaire n'est pas clairement désigné. Lear s'adresse à ses filles lorsqu'il demande une preuve d'amour, mais il semble que ce soient ses gendres qui héritent véritablement des terres et du pouvoir. Lear s'adresse d'abord à ses deux gendres (I.i.40-41) puisque les parts du royaume font office de dot. C'est donc l'ambiguité du statut juridique d'une dot qui introduit le doute sur le véritable récipiendaire de la cession : c'est une somme attribuée à une fille, mais destinée à devenir la propriété de son mari ${ }^{4}$. Ce flottement continue lorsque Lear s'adresse à ses filles pour lancer le test d'amour (I.i.48). Il s'agit bien, pour elles, de participer activement à la distribution de biens qui va avoir lieu, et il est donc suggéré qu'elles en sont les récipiendaires. Le langage est toujours hésitant quand Lear prononce le transfert luimême, une fois que Goneril a réussi l'épreuve : « Of all these bounds [...] / We make thee lady. To thine and Albany's issues / Be this perpetual » (I.i.63-67). La formule suggère que c'est Goneril qui reçoit ces terres, et que les générations suivantes pourront en hériter par leur mère. De même pour Regan : « To thee and thine hereditary ever / Remain this ample third of our fair kingdom» (I.i.79-80). Le placement de «to thee » en première position suggère ici encore que c'est avant tout Regan qui hérite, et par elle ses enfants ; le mari n'est même pas mentionné.

6 Mais plus loin, après le rejet de sa cadette, Lear s'adresse une fois de plus à ses gendres et leur demande de se partager la part de Cordelia et de prendre les rênes du royaume :

Cornwall and Albany, With my two daughters' dowers, digest this third.

$[\ldots]$

I do invest you jointly with my power,

Pre-eminence and all the large effects

That troop with majesty. Ourself by monthly course,

With reservation of an hundred knights

By you to be sustained, shall our abode

Make with you by due turn; only we shall retain

The name, and all th'addition to a king: the sway,

Revenue, execution of the rest, 
Beloved sons, be yours; which to confirm,

This coronet part between you.

I.i.128-139

La tirade est encadrée par des adresses aux gendres, elle leur est donc probablement destinée en totalité. La dot n'est plus transmise aux femmes, mais aux hommes, car la formule " with my two daughters' dowers, digest " suggère que les dots sont comprises dans la quantité de biens que les gendres doivent absorber. Ce sont eux qui ont l'obligation d'héberger Lear, ce qui est d'autant plus étonnant que, par la suite, il reprochera surtout à ses filles de ne pas subvenir à ses besoins. Lear termine sur un transfert de pouvoir et de revenus aux ducs, symbolisé par la transmission de la couronne aux gendres.

7 Comment expliquer cette double ambiguïté concernant le transfert de la scène d'exposition : la nature de la cession n'est pas claire (échange ou don); il y a un flou sur le récipiendaire (les filles ou les gendres) ? Les travaux de l'anthropologue Alain Testart permettent d'éclairer le premier problème. Il propose la définition suivante : « le don est la cession d'un bien qui implique la renonciation à tout droit sur ce bien ainsi qu'à tout droit qui pourrait émaner de cette cession, en particulier celui d'exiger quoi que ce soit en contrepartie ${ }^{5}$ ». C'est ce dernier point qui lui permet d'établir une différence entre le don et toutes les autres formes d'échange. En effet, s'il y a parfois une contrepartie dans le don (le « contre-don »), son obtention est affaire de codes moraux et sociaux ${ }^{6}$, et donc non garantie. En revanche, dans le cas de l'échange, l'obtention de la contrepartie est assurée par la loi. $\mathrm{Vu}$ dans cette perspective, le transfert opéré par Lear n'est manifestement pas un don, car la contrepartie est clairement exigée et attendue ; il s'agit plutôt d'un échange.

8 Si l'échange est fondé sur une obligation juridique de rendre une contrepartie, le transfert que cherche à effectuer Lear pose néanmoins problème. Dans une monarchie, la source de la loi est le monarque. Lear ne peut garantir l'exécution d'un échange convenu avec d'autres que s'il est roi. Or, l'échange consiste justement à abandonner ce rôle. Le transfert de propriété est à la fois un acte exprimant la souveraineté, où le roi dispose de son royaume à sa guise, et un geste qui annonce son impuissance et sa fragilité. Lear ne semble pas se fier à l'obligation légale de rendre contenue dans ce contrat oral qu'il passe avec la génération suivante au moment où il renonce au pouvoir. C'est ainsi que Lear se tourne vers un autre registre : l'obligation morale, ou sociale, de rendre, contenue dans le don. Katharine Eisaman Maus relève cette différence entre les domaines du don et de l'échange : "Rather like the unstrained mercy or the honorable liberality of The Merchant of Venice, gratitude cannot, apparently, be enjoined by force of positive law but only by the internalized dictates of duty, honor, and filial affection ${ }^{7} »$.

Le flottement constaté entre les registres de l'échange et du don est ainsi volontairement cultivé par Lear, qui veut s'assurer sa contrepartie par l'obligation sociale du don, en lieu et place de l'exigibilité juridique de l'échange à laquelle il renonce par l'abdication même. Or, ce type d'obligation sociale et morale contenue dans le don est d'autant plus forte qu'elle s'applique à des parents du sang: les filles, et non les gendres. Dès lors que les courtisans ont cessé d'être ses sujets, Lear ne peut exiger un contre-don que de ses enfants biologiques. Leur loyauté lui est garantie non par leur statut de sujet, mais par leur statut de filles. Le lien de parenté apparaît donc comme identique à un lien d'obligation : en tant que père, Lear a tout donné à ses filles, et réclame maintenant son dû. 
Lear exagère d'ailleurs volontairement le poids de cette double obligation, née à la fois du lien de parenté et du don. La cession très clairement décrite et cernée de la scène d'exposition se transforme, au fil de la pièce, en une dette généralisée que les filles auraient contractée envers leur père, marquée par « all » : « 0 , Regan, Goneril, / Your old, kind father, whose frank heart gave you all » (III.iv.19-20) ${ }^{8}$. Quel est donc ce " tout »? Cela comprend, bien sûr, le royaume, mais cela semble aussi englober une totalité mal définie : une vie aisée, une éducation peut-être ; en un mot, toute une vie de dévotion paternelle. Dès lors, à la totalité de ce « all » que Lear a donnée à ses filles, un contre-don total doit répondre. En créant une dette, une obligation totale, Lear crée le devoir de "tout» rendre, une obéissance absolue qu'il ne peut demander qu'à ses filles. Après avoir essuyé plusieurs refus de la part de Goneril au deuxième acte, Lear se tourne vers Regan pour obtenir son contre-don :

Thou [Regan] better knowst

The offices of nature, bond of childhood,

Effects of courtesy, dues of gratitude.

Thy half o'the kingdom hast thou not forgot, Wherein I thee endowed.

II.ii.366-370

Lear n'hésite pas à rappeler à sa fille toutes les choses qu'il lui a cédées pendant la cérémonie du premier acte. Mais il ajoute un autre registre: Regan serait obligée de rendre, non seulement à cause de la dette objective, matérielle, qui résulte de la cession du royaume, mais aussi à cause d'une obligation bien plus vague. «Effects of courtesy, dues of gratitude » fait référence à cette obligation sociale et morale de rendre un don donné. Une fois de plus, Lear se place sur le terrain du don, espérant que Regan s'acquittera de son obligation par simples bonnes mœurs. Enfin, Lear ajoute une troisième obligation : celle que les enfants doivent à leur père («bond of childhood»). Le terme "bond ", qui revient si souvent dans la pièce' ${ }^{9}$ exprime parfaitement ce lien entre parents et enfants qui serait créé par le don de ce « tout » que les premiers font aux derniers. Lear ne pouvant s'appuyer sur l'exigibilité juridique d'un «bond» au sens financier du terme après son abdication, il tente de faire appel à l'obligation de rendre contenue dans le «bond ", au sens d'obligation sociale, qui l'unit à ses filles ${ }^{10}$.

\section{«Beyond what can be valued ": Goneril et Regan ou la liberté moderne}

11 Comment, dans ce contexte, la jeune génération définit-elles ses devoirs face au père ? La réaction est évidemment différente pour Goneril et Regan d'un côté, et Cordelia de l'autre. Pendant la cérémonie du premier acte, Goneril et Regan, ayant bien compris que la simple rhétorique suffit à marquer l'obligation et la dette que Lear souhaite voir reconnues, cultivent elles aussi l'ambiguïté entre registres du don et de l'échange déjà mise en place par leur père. Voici comment s'exprime Goneril :

Sir, I do love you more than word can wield the matter;

Dearer than eyesight, space and liberty,

Beyond what can be valued, rich or rare,

No less than life, with grace, health, beauty, honour.

As much as child e'er loved, or father found,

A love that makes breath poor and speech unable,

Beyond all manner of so much I love you.

I.i.55-61 
Goneril reprend le discours de son père par son emploi de comparatifs. L'amour (ou, pour rester dans la logique de la pièce, le sens de l'obligation) - exprimé par Goneril est comparable, donc mesurable et quantifiable. La tirade peut ainsi se lire comme une allusion indirecte à l'échange qui est au centre du premier acte. Néanmoins, les allusions métatextuelles de Goneril à sa propre rhétorique révèlent le mode de fonctionnement qui caractérise son discours. D'après ce qu'elle dit, son amour est plus grand que les mots mêmes qui l'expriment. Cela peut paraître étonnant dans un discours si bien construit ; il semble que les mots soient parfaitement à même d'exprimer son amour. Ces phrases sont plutôt à prendre sur un mode proche de la prétérition : Goneril annonce qu'elle ne pourra pas s'exprimer convenablement pour, en fin de compte, réussir à très bien s'exprimer. Ce mode de fonctionnement s'étend à l'ensemble de son discours. Si on lit les nombreux comparatifs, non pas d'un point de vue grammatical (où ils indiquent évidemment une comparaison, donc une quantification), mais d'un point de vue sémantique, ils signifient tous que cet amour dépasse tous les points de comparaison qu'on pourrait lui appliquer il est incommensurable (« beyond»).

13 La négation de la quantification fait basculer le discours de Goneril dans le registre de l'incommensurable, du don infini. Puisque le père ne réclame que des mots, les filles peuvent lui rembourser son dû en mots; que la monnaie d'échange n'ait pas de valeur n'empêche pas pour l'instant l'échange d'être validé et permet à Goneril de fournir un contre-don inépuisable, car indénombrable. Il s'agit précisément de cette dette totale, absolue, que Lear invoquait face à ses filles : Goneril semble la reconnaître, et rendre une contrepartie (pour l'instant purement rhétorique) elle aussi infinie. Nous sommes tombés dans le registre du don, avec son obligation sociale et morale de rendre. Nous sommes donc face à une rhétorique à la fois de la quantification et de l'incommensurable qui dissimule et révèle l'échange que Lear propose. La prétérition apparaît alors comme l'embrayeur rhétorique qui permet de dire une chose et son contraire à la fois, de maintenir présents dans la scène les registres de l'échange et du don en même temps.

Les mêmes ambiguïtés se retrouvent dans le discours de Regan (I.i.69-76). Une fois de plus, nous trouvons l'idée de comparaison qui rend l'amour mesurable et attire l'attention sur les marques de quantité. Mais, dans la même tirade, Regan professe qu'elle dépasse encore sa sœur, rendant la comparaison caduque et entrant elle aussi dans le domaine de l'incommensurable. La stratégie rhétorique est donc la même que celle de Goneril.

L'emploi de ces deux registres permet aux sœurs aînées de rassurer leur père : il aura son dû, quel que soit le plan sur lequel on se place, celui de l'échange quantifiable (un certain nombre d'hectares contre un certain nombre de mots) ou celui du don illimité (l'amour illimité de leur père contre des mots indénombrables). Mais cette ambiguïté construit également une ironie dramatique. Si le langage de l'échange est présent dans cette scène, c'est qu'il annonce déjà que les sœurs refuseront, par la suite, la mécanique du don établie par Lear en refusant de reconnaitre leur obligation sociale et morale envers lui, la remplaçant par un échange de biens clairement dénombrés. La logique de Lear repose sur un échange de tout («all») contre tout; le choc sera celui du «nothing» en retour du «all ».

Les aînées ayant rempli leur part du contrat en participant au test d'amour, après n'avoir « donné » que des mots à leur père, elles reçoivent leur part du royaume en retour. Mais, comme le fait remarquer Testart, « un paiement acquitte une dette ; il engendre un droit, 
ou annule celui (l'obligation) que d'autres avaient sur vous ${ }^{11} »$. Selon la logique de l'échange adoptée par les filles, les obligations mutuelles que se doivent père et filles sont ainsi annulées, la dette payée, les liens personnels rompus. Au crescendo de la rhétorique dans la scène d'exposition se substitue plus tard un decrescendo dans le nombre de chevaliers que les filles veulent bien accorder à leur père (II.ii.377-475). Une fois la mascarade d'un amour prétendument incommensurable et gratuit terminée, il devient possible de nommer les quantités, et, partant, de les réduire. Cette diminution progressive jusqu'à zéro, possible dans le cadre de la logique de l'échange, va donc jusqu'à annuler le don même et les obligations qu'il implique.

Les aînées déploient ainsi une stratégie qui vise à les faire sortir de la mécanique établie par leur père, et qui les mettrait pour toujours dans l'obligation de rembourser éternellement leur dette. Le don met en effet le récipiendaire dans une position d'«obligé », l'endette, voire l'humilie et le domine. Ainsi émerge ce que les anthropologues nomment la "tyrannie du $\operatorname{don}^{12} »$ : le sentiment douloureux d'être endetté et de ne pouvoir rendre, une obligation morale et sociale impossible à observer. La réaction des aînées consistant à réduire leur relation avec leur père à un contrat annulable à tout instant apparaît alors comme un moyen de se libérer de cette tyrannie. Comme le résume si bien l'anthropologue Jacques Godbout: «la liberté moderne est essentiellement l'absence de dette ${ }^{13} »$. Si l'obligation du don est étouffante, la volonté d'en sortir conduit ici à rompre entièrement la relation, avec toute la violence que cela suppose. Cette coercition du père face à ses filles doit se lire dans le contexte d'une pièce où Lear apparaît de façon générale comme un père possessif. La critique féministe a souvent souligné le fait que Lear ne semble pas pouvoir laisser Cordelia partir ; sa soif de contrôle s'apparenterait même, selon certaines lectures, à des tendances incestueuses ${ }^{14}$.

\section{« Love my father all » : Cordelia ou l'aporie du don}

C'est Cordelia qui tente d'attirer l'attention de son père sur le problème d'une obligation excessive face au père, plaidant pour un équilibre entre l'amour dû au père et celui dû au mari. Dès la scène d'ouverture, elle défend une juste mesure dans son amour pour Lear :

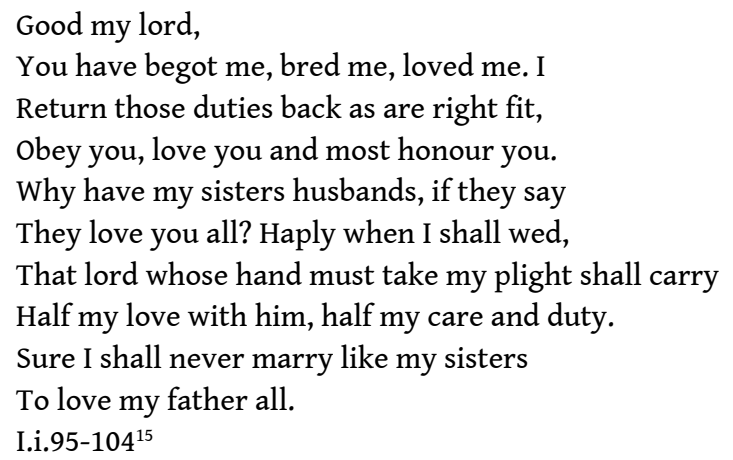

L'expression « return back » suggère que Cordelia reconnait à la fois le don qu'elle a reçu de son père et son obligation de rendre quelque chose. Mais ici encore, on constate une équivalence parfaite entre les obligations des uns et des autres, notamment marquée par le parallélisme des deux rythmes ternaires (vers 95 et 97). La logique de Lear fondée sur un don total est explicitement rejetée grâce au mécanisme de la question rhétorique et de la réponse. L'expression « love you all » apparaît d'abord dans un contexte interrogatif, puis une seconde fois dans la réponse que Cordelia fournit à sa propre question, mais accompagnée du négatif «never ». À ce double «all» du père, désormais écarté, elle 
substitue un double «half». Le dédoublement apporté par l'anaphore permet de souligner l'équilibre entre une obligation scindée en deux : moitié pour le père, moitié pour le mari. Contrairement à ce que demande Lear, Cordelia n'envisage pas d'obligation morale totale envers son père qui engagerait la personne de manière absolue. Le « $\mathrm{I}$ " placé en fin de vers, sous l'accent, atteste cette volonté de ne pas laisser s'effacer l'individu dans les liens qui l'unissent à d'autres.

Mais, comme l'a souvent souligné la critique, le contraste entre la Cordelia assez indépendante du premier acte et la Cordelia qui revient au quatrième acte est saisissant. Son mari est totalement absent; il n'est plus question d'un partage équilibré des obligations entre père et époux; elle mourra en prison. La mécanique du don est donc abolie, mais pas de la même manière que chez Goneril et Regan. Peu importe que Lear n'ait rien donné à sa fille au moment de la distribution des parts du royaume, elle lui donnera tout en retour : c'est « all » en retour de « nothing ${ }^{16}$ ». Finalement, le contre-don total que Cordelia rendra à son père, ce sera à la fois le don de sa personne, puisqu'elle mourra pour lui, et son " par-don » :

LEAR. I know you do not love me, for your sisters

Have, as I do remember, done me wrong.

You have some cause, they have not.

CORDELIA. No cause, no cause.

IV.vii.73-75

Lear suggère que Cordelia aurait toutes les raisons de ne plus aimer son père, mais elle répond «no cause ». La multiplication des structures négatives dans ces répliques n'est pas sans rappeler la réduction à zéro des obligations mutuelles entre parents et enfants qu'avaient envisagée les sœurs aînées. Mais la réduction à « nothing » n'a pas ici le même sens : elle prépare le contre-don total que Cordelia rendra à son père en échange de ce rien qu'elle a reçu, soulignant le caractère réellement infini du don de Cordelia.

La réponse en « no cause » peut également se lire comme un nouveau silence, en écho au silence de Cordelia pendant le test d'amour au début de la pièce. Jacques Derrida souligne en effet le paradoxe du pardon. Pour qu'il puisse y avoir pardon, la faute doit d'abord être nommée et reconnue. Mais par là même, la culpabilité est mise en avant: " pardonner, c'est consacrer le mal qu'on absout comme un mal inoubliable et impardonnable. [...] Le pardon accordé est aussi fautif que le pardon demandé, il avoue la faute ${ }^{17}$ ». D'où l'importance du silence, aussi bien dans le pardon que dans le don. Le don doit être tu, nié « car un don, pourrait-on dire, s'il se faisait reconnaître comme tel au grand jour, un don destiné à la reconnaissance s'annulerait aussitôt. Le don est le secret lui-même ${ }^{18}$ ». L'expression «no cause ", prise au pied de la lettre, n'exprime pas le pardon (même si, d'un point de vue pragmatique, c'est évidemment là son sens) ; elle nie en fait que Lear ait commis la moindre faute, et nie donc aussi la nécessité d'un pardon. C'est une manière de taire à la fois la faute et le «par-don » lui-même.

Quelle est donc la nature exacte de ce don de soi que fait Cordelia à son père à la fin de la pièce ? Pour Derrida, le don véritablement libre et gratuit n'existe pratiquement pas. Selon le philosophe, il faudrait une absence totale de récompense, il faudrait que le donateur ne s'attende même pas à en recevoir une, car ce serait déjà un calcul, et le geste ne serait plus véritablement désintéressé ${ }^{19}$ :

Dès l'instant où le don [...] se laisse effleurer par le calcul, dès lors qu'il compte avec

la connaissance ou la reconnaissance, il se laisse prendre dans la transaction : il échange, il donne en somme de la fausse monnaie, puisqu'il donne contre un salaire. [...] D'où la double « suppression de l'objet » [...] : dès qu'il calcule (à partir 
de la simple intention de donner comme telle, à partir du sens, de la connaissance et de la reconnaissance escomptée), le don supprime l'objet (du don). Il le nie en tant que tel. Pour éviter à tout prix cette négation ou cette destruction, il faudrait procéder à une autre suppression de l'objet : ne garder du don que le donner, l'acte et l'intention de donner, non le donné, qui au fond ne compte pas. Il faudrait donner sans savoir, sans connaissance et sans reconnaissance, sans remerciement : sans rien, en tout cas sans objet ${ }^{20}$.

Pourtant, le don de Cordelia à son père ressemble assez aux critères mis en place par Derrida pour définir un véritable don. Elle ne peut raisonnablement espérer une contrepartie, car son père n'est pas en mesure de lui donner quoi que ce soit. La véritable générosité, pour Derrida, se définit comme suit :

À quelle condition peut-il y avoir responsabilité ? À la condition que le Bien soit [...] le rapport à l'autre, une réponse à l'autre : expérience de la bonté personnelle et mouvement intentionnel. [...] À quelle condition y a-t-il bonté, au-delà du calcul ? À la condition que la bonté s'oublie elle-même, que le mouvement soit un mouvement de don qui renonce à soi, donc un mouvement d'amour infini ${ }^{21}$.

Le pardon de Cordelia est bien une renonciation à soi ; il est ce "mouvement d'amour infini » qui tend vers l'autre. La notion de responsabilité est essentielle dans la pensée de Derrida sur le don, car elle exige «qu'on réponde, en tant que soi-même et en tant que singularité irremplaçable, de ce qu'on fait, dit, donne ${ }^{22} »$. Ainsi, la responsabilité ne peut être que personnelle. Or, «faire l'expérience de la responsabilité, depuis la loi donnée, faire l'expérience de sa singularité absolue et appréhender sa propre mort, c'est la même expérience : la mort est bien ce que personne ne peut ni endurer ni affronter à ma place ${ }^{23}$ ». L'expérience de la responsabilité comme réponse à l'autre, comme mouvement vers l'autre, est donc aussi une expérience de la mort du moi :

Que veut dire en français donner la mort? Comment se donne-t-on la mort? Comment se la donne-t-on au sens où se donner la mort, c'est mourir en assumant la responsabilité de sa mort, se suicider mais aussi bien se sacrifier pour autrui, mourir pour l'autre, donc peut-être donner sa vie en se donnant la mort, en acceptant la mort donnée $[. . .]^{24}$ ?

Mais en même temps, la responsabilité suppose également une forme d'autonomie, celle du choix de ses actes, de ses paroles, car « ce souci de la mort, cet éveil qui veille sur la mort, cette conscience qui regarde la mort en face, est un autre nom de la liberté25 Ainsi Cordelia assume-t-elle sa responsabilité envers son père en lui venant en aide, en répondant à l'autre. Il s'agit bien d'un choix parfaitement libre, puisque rien ne l'y obligeait. Mais la responsabilité choisie librement, et qui consiste à faire ce don d'amour infini à son père, se terminera pour elle dans une mort réelle. Derrida envisage peut-être une mort dans un sens plus métaphorique - je prends conscience du fait que je suis prêt à donner, donner de moi, me sacrifier pour l'autre. Or, c'est bien ce risque ultime que prend Cordelia lorsqu'elle lance son armée contre ses sœurs, celui d'une défaite si totale qu'elle pourrait lui coûter la vie. Sa mort réelle, au cinquième acte, ne vient alors que dédoubler le sens qu'avait déjà son geste au quatrième acte : elle était prête à donner de soi, se donner pour son père. C'est un don marqué à la fois par une liberté et une responsabilité totales, un don de soi-même dans lequel le sujet accepte la possibilité de sa propre annihilation; le seul don de «all » dans une pièce où chacun cherche à donner le moins possible.

Mais c'est un don qui débouche sur une aporie: dans cette tragédie, le seul don véritablement désintéressé mène nécessairement à la mort. C'est ainsi que le décès de Cordelia peut aussi être lu dans une lumière moins positive, comme le fait notamment la 
critique féministe, car son sacrifice est ce qui permet à Lear de réaliser tous ses désirs les plus discutables. Il peut enfin garder sa fille, dont le mari a disparu. La perspective de passer le reste de ses jours en prison avec elle est source de bonheur, puisque désormais ils seront unis pour toujours (V.iii.8-19). Lear semble enfin réaliser son rêve : «I [...] thought to set my rest / On her kind nursery » (I.i.124-125). Cette fusion s'accompagne du silence de Cordelia, qui n'apparaîtra plus sur scène. Lear remarquera après sa mort : « Her voice was ever soft, / Gentle and low, an excellent thing in woman» (V.iii.270-271). Cordelia devient définitivement l'objet du fantasme de son père, et non plus un sujet autonome. La fille idéale semble être la fille qui ne parle que lorsque son père l'exige d'elle. Lynda Boose remarque : " the father who imagined that he "gave his daughters all" extracts from his daughter at the end of the play the same price he demanded in the opening scene - that she love her father all ${ }^{26} »$.

Si Derrida voit dans le silence l'une des conditions de possibilité du don, c'est justement le silence qui souligne également l'immensité de ce don qui tait la faute du père et coûte la vie à Cordelia. Goneril et Regan, qui manient si bien les mots, savent au contraire manipuler les liens d'obligation à leur avantage pour sauvegarder leur liberté. Mais cette absence de dette qu'elles arrachent de force annule tous les liens, notamment les liens de parenté, et finit par les annihiler elles-mêmes. Si la pièce montre une méconnaissance tragique du don et de son incroyable force chez les aînées, elle ne permet donc pas à la jeunesse d'entrevoir une existence autonome en dehors des liens du don.

\section{NOTES}

1. Cette idée est liée au concept de «tyrannie du don » en anthropologie, sur lequel nous reviendrons.

2. William Scott nous rappelle que le procédé est de toute façon illégal, puisque le royaume est inaliénable, notamment selon le droit médiéval (William Scott, « Contracts of Love and Affection: Lear, Old Age, and Kingship », Shakespeare Survey 55, 2002, 36-42, note 2 page 36) - s'agit-il d'un indice suggérant que la cession du royaume est plutôt affaire de don en famille qu'une affaire d'État pour Lear ? En revanche, il existe un procédé juridique à la Renaissance, à la disposition des roturiers, qui ressemble fortement à ce que Lear met en place ici: le "maintenance agreement ». Coppélia Kahn explique de quoi il s'agit : "In such contracts, children to whom parents deeded farm or workshop were legally bound to supply food, clothing, and shelter to their parents, even to the precise number of bushels of grain or yards of cloth. Thus the law put teeth into what was supposed to be natural kindness. Lear's contest of love in the first scene functions as a maintenance agreement in that he tries to bind his daughters, by giving them their inheritance while he is still alive, into caring for him " (Coppélia Kahn, «The Absent Mother in King Lear ", in Rewriting the Renaissance: The Discourse of Sexual Difference in Early Modern Europe, dir. Margaret W. Ferguson, Maureen Quilligan et Nancy J. Vickers, Chicago, University of Chicago Press, 1986, 33-49, p. 44-45). William Scott donne par ailleurs des exemples détaillés de ce genre de contrat, qui éclairent des passages de la pièce tels que celui où Lear réclame à ses filles : «I beg / That you'll vouchsafe me raiment, bed and food » (II.ii.344-345). Scott conclut : «[Lear] is making a sort of contract to buy future love » (Scott, op. cit., p. 38). 
3. Toutes les citations du Roi Lear sont tirées de : William Shakespeare, King Lear, éd. R.A. Foakes, The Arden Shakespeare, Third Series, Londres, 1997.

4. Lear établit un lien entre la cession de ses biens et les dots de ses filles, ce qui n'est pas totalement incongru à l'époque, puisque les «marriage settlements » contenaient souvent des clauses s'apparentant à un "maintenance agreement " pour les parents des mariés. Katharine Eisaman Maus relève elle aussi le problème du véritable récipiendaire des biens, puisqu'une dot, bien qu'attribuée à une fille (ici dans le cadre du test d'amour), devient la propriété de son mari selon la doctrine de " coverture » : "In Lear's particular family circumstances, the fact that two of his daughters are already married raises a further problem: the question of to whom the segmented realm is actually being conveyed. Lear begins the love-test by addressing Albany and Cornwall, and calling the kingdom he means to divide "our daughters' several dowers". In other words, he apparently assumes that the daughters' entitlement will pass to their husbands "under coverture", as property normally would in marriage, and therefore invests the husbands, not his daughters, with "my power, / Preeminence, and all the large effects / That troop with majesty" (130-132). [...] Kent and Gloucester [...] assume that the husbands of Goneril and Regan will be the de facto recipients of any territories granted to their wives" (Katharine Eisaman Maus, « Vagabond Kings: Entitlement and Distribution in 2 Henry VI and King Lear », in Being and Having in Shakespeare, Oxford, Oxford University Press, 2013, 99-132, p. 114). Mais le contexte juridique n'est pas si simple, car les exceptions à la « coverture » sont nombreuses. La première, analysée par Maus, concerne la famille royale : « it is still unclear if a regnant queen's husband - a son-inlaw rather than a "son of the blood" - is really a king in every respect » (p. 115). La question était bien connue des contemporains car le statut de Philippe d'Espagne, époux de la reine Marie, était pour le moins flou. Voici les conséquences pour la famille de Lear selon Maus : «Confronted by Albany with proof of her adultery with Edmund, Goneril informs her husband that he has no authority over her: "The laws are mine, not thine" (V.iii.157-158). [...] Goneril, her monstrousness aside, is possibly correct. [...] via her performance of the love test she has received sovereignty in her own person, not as a conduit to her husband » (p. 115). Par ailleurs, l'historienne Amy Louise Erickson analyse la pratique des « marriage settlements » de façon plus générale, concluant : «A marriage settlement was a legal evasion of the law of coverture and dower [...]. One made a marriage settlement in order to avoid the property law incidents which accompanied marriage. Both wills and marriage settlements have been described as deviations from custom, but especially in some segments of the population the deviation was so common as to have become custom itself » (Amy Louise Erickson, Women and Property in Early Modern England, Londres, Routledge, 1993, p. 100-101). Ainsi, la pratique du «separate estate» (aussi connue sous le nom de " pin-money ») était-elle déjà bien établie à la Renaissance. Erickson précise : "Conveyancing manuals throughout the early modern period demonstrate that the primary purpose of a marriage settlement was to preserve the wife's property rights. The sample forms of settlement presented incorporated any one or more of the following features: setting the amount of the wife's jointure; allowing her to make a will under coverture; obliging her husband to leave her worth so much money at his death; or binding him to pay portions to her children by a previous husband " (p. 104). Elle cite notamment le cas de Lady Anne Clifford sous le règne de Jacques $\mathrm{I}^{\mathrm{er}}$, qui refusa de se séparer de ses terres dans le Westmorland au profit de son mari (p. 111-112). Concluons donc que les ambiguités juridiques autour de la dot et de la «coverture » reflètent l'ambiguïté de Lear lui-même qui s'adresse tantôt à ses filles tantôt à ses gendres lors de la cession de biens.

5. Alain Testart, Critique du don : études sur la circulation non marchande, Paris, Syllepse, 2007, p. 19. La définition de Testart est issue d'une critique de l'œuvre de Marcel Mauss, l'un des premiers anthropologues à se pencher sur la question du don. Il constate un flou dans les termes utilisés par Mauss et il se propose d'affiner les catégories implicitement créées par ce dernier. Mauss est en effet ambigu quand il évoque « le caractère volontaire, pour ainsi dire, apparemment libre et 
gratuit, et cependant contraint et intéressé de ces prestations » (Marcel Mauss, « Essai sur le don. Forme et raison de l'échange dans les sociétés archaïques", L'année sociologique, 1923-1924, $30-186$, p. 33). Testart se propose ainsi se séparer plus nettement entre un geste gratuit et désintéressé d'une part, et un geste pour lequel un remboursement, une récompense, une contrepartie ou un contre-don, seraient exigibles, d'autre part. Cela l'amène d'ailleurs à qualifier d'« échange » certains des phénomènes que Mauss analyse sous le titre de « don ».

6. La question de la nature exacte de l'obligation dans le don a fait débat en anthropologie. Nous nous en tiendrons à la perspective de Testart, pour qui l'obligation de rendre naît du simple fait qu'une chose a été donnée et que le donataire, transformé en obligé, se sent lui-même dans l'obligation de rendre. L'obligation naît du sentiment d'une dette - peu importe, d'ailleurs, si le premier don avait été sollicité ou même voulu - dont on cherche à s'acquitter. Testart résume : «La force qui pousse à rendre est celle de l'obligation, quelle que soit sa nature, sociale, juridique ou seulement morale. Et obligare, faut-il rappeler, c'est se lier : la force qui pousse à rendre est celle du lien social» (Testart, op. cit., p. 195). C'est donc tout simplement la force du lien - aussi fugace soit-il, mais forcément «social »- unissant deux personnes qui échangent un objet qui donne toute sa puissance à cette obligation.

7. Maus, op. cit., p. 124.

8. Le mot «all » revient souvent dans la bouche de Lear, par exemple dans les questions que Lear adresse à Edgar déguisé en mendiant, dont la pauvreté s'expliquerait par le fait qu'il aurait tout donné à ses filles (III.iv). Le Fou répète également cette idée.

9. Dans tous les contextes où le mot «bond " apparaît dans la pièce, il exprime le lien qui unit un père à ses enfants, même s'il n'a pas le même sens pour chaque génération. C'est particulièrement évident dans la relation entre Gloucester et ses fils. Edmond manie ce concept avec brio, l'employant toujours pour créer l'ironie dramatique. Il prétend avoir rappelé à l'ordre son frère soi-disant déloyal : « Spoke with how manifold and strong a bond / The child was bound to the father» (II.i.47-48). Dans la fausse lettre censée avoir été écrite par Edgar, Edmond exprime en fait ses propres idées. Ici, «bond» s'est transformé en "bondage», synonyme de «tyranny " : "I begin to find an idle and fond bondage in the oppression of aged tyranny, who sways not as it hath power, but as it is suffered » (I.ii.49-51). Pour cet enfant, le lien entre père et fils est trop fort, au point de rendre une rupture par la force nécessaire. Ajoutons que, dans la langue anglaise, «bond» désigne également l'effet de commerce qui promet le paiement d'une somme d'argent - sens au cœur du Marchand de Venise - et insiste ainsi sur l'idée d'un lien entre deux personnes fondé sur la dette et l'obligation de rembourser.

10. L'expression "offices of nature" pourrait faire référence à la loi naturelle, notion sur laquelle s'appuient plusieurs personnages, notamment Gloucester et Lear, pour insister sur les liens qui les unissent à leurs enfants: le lien entre générations serait «naturel» et donc inévitable; les enfants qui se rebellent contre ce lien seraient alors « contre-nature ». D'où les reproches aux enfants concernant leur soi-disant manque de gratitude ou d'allégeance, notions fondées elles aussi sur des liens soi-disant naturels. Mais cet appel à la loi naturelle pourrait aussi se lire autrement. Il s'agit pour les pères de présenter comme un fait évident, un "donné " inéluctable, quelque chose qui relève en fait de la convention sociale, à savoir les liens d'obligation (dans tous les sens du terme) qui unissent les générations. Peu importe, donc, sur quel mode cette obligation est déclinée (gratitude, allégeance, affection, amour...), la conséquence est la même : il s'agit de forcer les enfants à obéir à leurs pères.

11. Testart, op. cit., p. 21.

12. L'anthropologue Jacques Godbout fait remonter l'expression au livre suivant, même si elle s'est désormais établie dans cette discipline: Renée Fox et Judith Swazey, Spare Parts. Organ Replacement in American Society, New York, Oxford University Press, 1992. Voir Jacques Godbout, Le don, la dette et l'identité: homo donator versus homo œconomicus, Paris, Le Bord de l'Eau, 2013, p. 139. Godbout soulève par ailleurs la question suivante : l'obligation de retour a-t-elle une force 
particulière dans les dons qu'il nomme « intenses ", comme l'héritage (Ibid., p. 135) ? Le Roi Lear tendrait à confirmer que c'est bien le cas.

13. Ibid., p. 47 (c'est Godbout qui souligne).

14. Voir notamment les analyses suivantes. Janet Adelman, "Suffocating Mothers in King Lear ", Suffocating Mothers. Fantasies of Maternal Origin in Shakespeare's Plays, Hamlet to The Tempest, New York, Routledge, 1992, 103-129 ; Lynda Boose, « The Father and the Bride in Shakespeare », PMLA 97.3, 1982, 325-347; Kahn, op. cit.

15. Le dernier vers « To love my father all » n'est présent que dans le Quarto publié à Londres en 1608.

16. Lear avait lui-même insisté sur le «nothing " donné à Cordelia au premier acte : «nothing will come of nothing. Speak again » (I.i.90) ; «there she stands / [...] with our displeasure pieced / And nothing more » (I.i.198-201) ; et, en réponse à la demande du duc de Bourgogne qui veut savoir si elle aura une dot, « Nothing. I have sworn » (I.i.247).

17. Jacques Derrida, Donner la mort, Paris, Galilée, 1999, p. 182.

18. Ibid., p. 50. Derrida explique ainsi, par exemple, l'injonction biblique de donner sans qu'une main sache ce que fait l'autre (Ibid., p. 145).

19. Notons qu'il existe sur ce point un différend marqué entre Derrida et de nombreux anthropologues. Alain Testart par exemple écrit : « le contre-don n'annule pas le don : il rend nul le solde comptable [...]. Il a seulement pour effet d'annuler la dette (morale), mais il n'annule pas le geste du don. Ni le fait qu'il y ait eu don. Ni même la reconnaissance que l'on peut avoir vis-àvis de quelqu'un du fait qu'il vous a donné » (Testart, op. cit., p. 233 [c'est Testart qui souligne]).

20. Derrida, op. cit., p. 152-153 (c'est Derrida qui souligne). D'un point de vue étymologique, «merci » veut dire « prix, salaire, récompense »; le remerciement apparaît ainsi comme salaire du don, donc comme calcul.

21. Ibid., p. 76-77.

22. Ibid., p. 78.

23. Ibid., p. 64.

24. Ibid., p. 26-27 (c'est Derrida qui souligne).

25. Ibid., p. 33.

26. Boose, op. cit., p. 335.

\section{RÉSUMÉS}

Si les liens familiaux, et donc intergénérationnels, sont un thème dominant de l'œuvre shakespearienne, comment la vision de la jeunesse évolue-t-elle dans les pièces de la maturité ? Le Roi Lear met explicitement en scène un affrontement entre générations jeunes et âgées, qui peut se lire comme un conflit autour de la méconnaissance tragique du don. En transmettant son royaume à ses filles, le patriarche met en place un mécanisme selon lequel une obligation absolue de rendre doit répondre à la totalité d'un don absolu («I gave you all »). Face à cette dette quasiimpossible à rembourser, Goneril et Regan ne trouveront d'autre issue que d'annuler le contrat, exposant ainsi la jeunesse à l'accusation d'ingratitude. Ce qui est en jeu, ce sont bien les droits et devoirs réciproques que se doivent les générations. Quelle est la légitimité d'une jeunesse qui refuse le poids de l'obligation imposée par le don des aînés? 
If familial - and therefore intergenerational - bonds are a dominant theme of Shakespeare's work, how does the representation of youth evolve in the late plays? King Lear explicitly highlights the rivalry between older and younger generations, which can be read as a conflict around a tragic misunderstanding of gift-giving. By bequeathing his kingdom to his daughters, the patriarch creates a mechanism by which an absolute obligation to return the gift must mirror the totality of an absolute gift («I gave you all »). Faced with a debt that is almost impossible to repay, Goneril and Regan will find no other solution but to annul the contract, introducing the theme of the scandalous ingratitude of youth. The rights and duties the generations owe one another are at stake. How legitimate is a young generation who reject the burdensome obligations imposed by the gifts of the old?

\section{INDEX}

Keywords : debt, forgiveness, gift-giving, King Lear

Mots-clés : Derrida Jacques, dette, don, obligation, pardon, Roi Lear (le), Testart Alain

\section{AUTEUR}

\section{ANNE-KATHRIN MARQUARDT}

Professeur en classes préparatoires littéraires, Lycée Joliot-Curie, Nanterre 